\title{
A HIGHER-DIMENSIONAL GENERALIZATION OF MUMFORD'S RATIONAL PULLBACK FOR WEIL DIVISORS
}

\author{
STEFAN SCHRÖER
}

17 July 2018

\begin{abstract}
Mumford defined a rational pullback for Weil divisors on normal surfaces, which is linear, respects effectivity, and satisfies the projection formula. In higher dimensions, the existence of small resolutions of singularities precludes such general results. We single out a higher-dimensional situation that resembles the surface case and show for it that a rational pullback for Weil divisors exists, which is also linear, respects effectivity, and satisfies the projection formula.
\end{abstract}

\section{Contents}

Introduction 1

1. Rational pullback 2

2. Invertible M-matrices 5

References $\quad 8$

\section{INTRODUCTION}

An important result for the theory of algebraic surfaces is Mumford's rational pullback [9]: Let $f: X \rightarrow S$ is the resolution of singularities for a normal surface $S$. Then the pullback for Cartier divisors extends to a unique map

$$
f^{*}: Z^{1}(S) \longrightarrow Z^{1}(X)_{\mathbb{Q}}=Z^{1}(X) \otimes_{\mathbb{Z}} \mathbb{Q}
$$

for Weil divisors that retains the usual properties, namely the map $f^{*}$ is linear, respects effectivity, and satisfies the projection formula. The existence of this pullback relies on the fact that the intersection matrix $\Phi=\left(E_{i} \cdot E_{j}\right)$ for the exceptional divisors $E_{i} \subset X$ is negative-definite, with strictly negative entries along the diagonal and positive off-diagonal entries. Many definitions and results for smooth surfaces extend to normal surfaces, by using Mumford's rational pullback. For example, the $\mathbb{Z}$-valued intersection form for Cartier divisors on proper surfaces extends to a $\mathbb{Q}$-valued intersection form for Weil divisors.

Despite the importance of a rational pullback, in particular for canonical divisors in the minimal model program, there have been little attempts to generalize Mumford's rational pullback to higher dimensions. Indeed, the existence of small resolutions of singularities in dimension $d \geq 3$ precludes unconditional results. Nevertheless, de Fernex and Hacon [5] succeeded to construct a real-valued pullback using valuation theory and asymptotic behavior in a surprising way. In this general

2010 Mathematics Subject Classification. 14E05, 14C20, 14A15. 
set-up, however, it is not so clear when linearity holds, effectivity is preserved and the projection formula remains true. The main goal of this paper is to single out a higher-dimensional situation that sufficiently resembles the surface case and that yields a rational pullback with these three properties.

We work in the following general set-up: Let $S$ be the spectrum of a noetherian ring $R$ that is local and normal of dimension $d \geq 2$, and $f: X \rightarrow S$ be a proper birational morphism with $X$ integral and normal. We do not require a ground field, but for the sake of exposition we assume that $R$ is excellent. Our main result is:

Theorem. (See Thm. 1.2) The morphism $f: X \rightarrow S$ admits a rational pullback provided the following three conditions holds:

(i) All local rings $\mathscr{O}_{X, x}$ are $\mathbb{Q}$-factorial.

(ii) The exceptional locus $\operatorname{Exc}(X / R)$ and the closed fiber $f^{-1}(z)$ coincide as closed sets, and this is equidimensional of dimension $d-1$.

(iii) Its irreducible components $E_{1}, \ldots, E_{r}$ have Picard number $\rho=1$.

Note that the three assumptions hold in dimension $d=2$ for any resolution of singularities. It is easy to produce example in higher dimension, by contracting suitable Cartier divisors.

The key idea for the above result is to work with the non-symmetric square matrix $\Phi=\left(E_{i} \cdot C_{j}\right)$, where $C_{j} \subset E_{j}$ are chosen curves. The crucial point is to establish that $A=-{ }^{t} \Phi$ is an invertible $M$-matrix, a very useful notion from linear algebra going back to Minkowski that generalizes positive-definiteness for symmetric matrices to arbitrary square matrices. The theory of invertible M-matrices is widespread in applied mathematics, but perhaps not so well-known in pure mathematics. Our approach relies on some recent contractibility results in [11], which in turn are based on Cutkosky's study of graded linear system and his generalization of big invertible sheaves to non-integral schemes [4].

The paper is organized as follows: In the first section, we recall Mumford's rational pullback for surfaces, discuss the problem of extending it to higher dimensions, and state our main result. The second section contains the proofs.

Acknowledgement. This research was conducted in the framework of the research training group GRK 2240: Algebro-geometric Methods in Algebra, Arithmetic and Topology, which is funded by the DFG.

\section{Rational PULlbaCK}

Let $R$ be a local noetherian ring that is normal of dimension $d \geq 2$, with residue field $k=R / \mathfrak{m}_{R}$, spectrum $S$ and closed point $z \in S$. For the sake of exposition, I also assume that the ring $R$ is excellent. Let $Z^{1}(S)$ be the group of Weil Divisors, which is the free abelian group generated by the prime divisors $D \subset S$. This is an ordered group in the sense of [3], Chapter VI, where the positive elements $D \geq 0$ are the effective divisors $D \subset X$. The same applies to the group of $\mathbb{Q}$-divisors $Z^{1}(S)_{\mathbb{Q}}=Z^{1}(S) \otimes \mathbb{Q}$ and the subgroup of Cartier divisors Cart $(S)$. Note that throughout we use the term "positive" in Bourbaki's sense $x \geq 0$, and "strictly positive" for $x>0$. 
Let $f: X \rightarrow S$ be a proper birational morphism, where $X$ is integral and normal. For each point $s \in S$, the fiber $f^{-1}(s)$ is a proper scheme over the field $\kappa(s)$. Its one-dimensional closed subschemes are called vertical curves. For each invertible sheaf $\mathscr{L} \in \operatorname{Pic}(X)$ and each vertical curve $C \subset f^{-1}(s), s \in S$ we get an intersection number $(\mathscr{L} \cdot C)=\chi\left(\mathscr{L}_{C}\right)-\chi\left(\mathscr{O}_{C}\right)$, where the Euler characteristics are computed over the residue field $\kappa(s)$.

For each effective Cartier divisor $D \subset S$, the subscheme $f^{-1}(D) \subset X$ remains an effective Cartier divisor, because $X, S$ are integral and $f: X \rightarrow S$ is dominant. This fact yields a pullback homomorphism for Cartier divisors

$$
f^{*}: \operatorname{Cart}(S) \longrightarrow \operatorname{Cart}(X) \subset Z^{1}(X),
$$

which is linear, increasing, and satisfies the projection formula. The latter means $(\mathscr{L} \cdot C)=0$ for each vertical curve $C \subset f^{-1}(s), s \in S$. We seek to extend (1) to a rational pullback $f^{*}: Z^{1}(S) \rightarrow Z^{1}(X)_{\mathbb{Q}}$ that is also linear, increasing and satisfies the projection formula. Such an extension exists a priori on the subgroup of $\mathbb{Q}$ Cartier divisor. The crux here is that we want to extend further, without making any assumption on the class group $\mathrm{Cl}(R)=Z^{1}(S) / \operatorname{Cart}(S)$. However, to make sense of the intersection numbers in the projection formula, we will usually assume that the local rings $\mathscr{O}_{X, x}$ are $\mathbb{Q}$-factorial, that is, the abelian groups $\operatorname{Cl}\left(\mathscr{O}_{X, x}\right)$ are torsion groups.

Now suppose we are in dimension $d=2$. Then the exceptional locus $\operatorname{Exc}(X / R)=$ $\operatorname{Supp}\left(\Omega_{X / R}^{1}\right)$ coincides with the closed fiber $f^{-1}(z)$, and the underlying reduced closed subscheme $E \subset X$ is equidimensional, of $\operatorname{dimension} \operatorname{dim}(E)=1$. Decompose $E=E_{1}+\ldots+E_{r}$ into irreducible components. Under the assumption that all local rings $\mathscr{O}_{X, x}$ are $\mathbb{Q}$-factorial, we get a $\mathbb{Q}$-valued intersection matrix $\Phi=\left(E_{i} \cdot E_{j}\right)$. This matrix is symmetric and negative-definite, an observation going back to Mumford [9], Artin [1] and Deligne ([6], Exposé X, Corollary 1.9), in various forms of generality. Mumford used this to define the rational pullback $f^{*}: Z^{1}(S) \rightarrow Z^{1}(X)_{\mathbb{Q}}$ as follows: For each prime divisor $D \subset S$, the strict transform $D^{\prime} \subset X$ yields certain intersection numbers $\left(D^{\prime} \cdot E_{i}\right) \geq 0$. Since $\Phi$ is invertible, there is are unique rational numbers $m_{1} / n, \ldots, m_{r} / n$ with $\left(n D^{\prime} \cdot E_{j}\right)=-\left(\sum m_{i} E_{i} \cdot E_{j}\right)$, for each $1 \leq j \leq r$. Mumford sets

$$
f^{*}(D)=D^{\prime}+\frac{1}{n} \sum m_{i} E_{i}
$$

and extends by linearity ([9], Section II (b)). By construction, the projection formula $\left(f^{*}(D) \cdot E_{j}\right)=0$ holds. A non-trivial fact from linear algebra ensures that all entries of $\Phi^{-1}$ are negative, hence $m_{i} / n \geq 0$, so the rational pullback preserves effectivity.

In the general situation $d \geq 2$, and write $E_{1}, \ldots, E_{r} \subset \operatorname{Exc}(X / R)$ for the irreducible components of dimension $\operatorname{dim}\left(E_{i}\right)=d-1$. This are precisely those prime divisors on $X$ whose images on $S$ cease to be a divisor. The existence of a rational pullback can be seen as a problem in linear programming:

Definition 1.1. Suppose that all local rings $\mathscr{O}_{X, x}$ are $\mathbb{Q}$-factorial. We say that $f: X \rightarrow S$ admits a rational pullback if for each $\mathscr{L} \in \operatorname{Pic}(X)$ having a global section that does not vanish along $E_{1}, \ldots, E_{r}$, there are unique rational number $m_{1} / n, \ldots, m_{r} / n \in \mathbb{Q}_{\geq 0}$ so that $\left(\mathscr{L}^{\otimes n} \cdot C\right)=-\left(\sum m_{i} E_{i} \cdot C\right)$ for all vertical curves $C \subset f^{-1}(s), s \in S$. 
Indeed, we then define the homomorphism $f^{*}: Z^{1}(S) \rightarrow Z^{1}(X)_{\mathbb{Q}}$ by the formula

$$
f^{*}(D)=D^{\prime}+\frac{1}{n n^{\prime}} \sum m_{i} E_{i}
$$

where $D$ is a prime divisor, $D^{\prime}$ is its strict transform, $n^{\prime}>0$ is an integer such that $n^{\prime} D^{\prime}$ is Cartier, and the coefficients $m_{i} / n$ arise from the invertible sheaf $\mathscr{L}=$ $\mathscr{O}_{X}\left(n^{\prime} D^{\prime}\right)$. Clearly, this does not depend on the choice of $n^{\prime}$, and the map is linear, increasing, and satisfies the projection formula. If $D \subset S$ is an effective Cartier divisor, then $f^{-1}(D)=D^{\prime}+\sum m_{i} E_{i}$ is numerically trivial on all vertical curves. Hence $f^{-1}\left(n^{\prime} D\right)$ coincides with the rational pullback $f^{*}\left(n^{\prime} D\right)$, whenver $n^{\prime} D^{\prime} \subset X$ is Cartier. Since $Z^{1}(X)_{\mathbb{Q}}$ is torsion-free, we already have $f^{-1}(D)=f^{*}(D)$. It follows that the rational pullback extends the usual pullback for Cartier divisors. Conversely, if such a map $f^{*}: Z^{1}(S) \rightarrow Z^{1}(X)_{\mathbb{Q}}$ exists, (2) yields the desired coefficients in Definition 1.1, by setting $\mathscr{L}=\mathscr{O}_{X}\left(n^{\prime} D^{\prime}\right)$.

It seems difficult to verify directly that a morphism $f: X \rightarrow S$ admits a rational pullback. The main result of this paper is the following criterion, whose proof will occupy the second section:

Theorem 1.2. The morphism $f: X \rightarrow S$ admits a rational pullback provided the following three conditions holds:

(i) All local rings $\mathscr{O}_{X, x}$ are $\mathbb{Q}$-factorial.

(ii) The exceptional locus $\operatorname{Exc}(X / R)$ and the closed fiber $f^{-1}(z)$ coincide as closed sets, and this is equidimensional of dimension $d-1$.

(iii) Its irreducible components $E_{1}, \ldots, E_{r}$ have Picard number $\rho=1$.

Each exceptional divisor $Y=E_{i}$ is a proper $k$-scheme. Let $Z_{1}(Y)$ be the free abelian group generated by the integral curves $C \subset Y$, and $\operatorname{Pic}(Y) \times Z_{1}(Y) \rightarrow \mathbb{Z}$ be the ensuing intersection pairing. The radical on the left is $\operatorname{Pic}^{\tau}(Y)$, the group of numerically trivial invertible sheaves. By Finiteness of the Base, the residue class group $N^{1}(Y)=\operatorname{Pic}(Y) / \operatorname{Pic}^{\tau}(Y)$ is finitely generated. Being torsion-free, it must be free. Its rank $\rho \geq 0$ is called the Picard number. Let $N^{1}(Y) \times N_{1}(Y) \rightarrow \mathbb{Z}$ be the induced non-degenerate pairing. Then also $N_{1}(Y)$ is finitely generated and free, of rank $\rho \geq 0$.

In dimension $d=2$, we have $N_{1}\left(E_{i}\right)=\mathbb{Z}$, and the conditions of the theorem are automatically satisfied for any resolution of singularities $f: X \rightarrow S$. We thus recover Mumford's rational pullback. Actually, it suffices to assume that the local rings $\mathscr{O}_{X, x}$ are $\mathbb{Q}$-factorial.

It is not difficult to construct proper birational morphisms $f: X \rightarrow S$ in arbitrary dimension $d \geq 2$ for which our result applies: Let $A$ be an excellent discrete valuation ring, with residue field $k=A / \mathfrak{m}_{A}$, and consider any projective flat $A$-scheme $Y$ whose closed fiber $Y \otimes_{A} k$ is smooth, with Picard number $\rho=1$. According to [11], Proposition 1.6, there is an effective Cartier divisor $Z \subset Y \otimes_{A} k$ so that on the blowing-up $\varphi: \mathrm{Bl}_{Z}(Y) \rightarrow Y$, the strict transform $E$ of the closed fiber $Y \otimes_{A} k$ admits a contraction $\varphi^{\prime}: \mathrm{Bl}_{Z}(Y) \rightarrow Y^{\prime}$ to some projective $A$-scheme $Y^{\prime}$. Such a construction resembles the elementary transformations for projective bundles, and was already used in [10] for surfaces. Using Bertini, one can arrange things that $Z$ is smooth, hence the total space $\mathrm{Bl}_{Z}(Y)$ is regular. The image $z=\varphi^{\prime}(E)$ is a closed point. Let $R=\mathscr{O}_{Y^{\prime}, z}$ be the resulting local ring. With $S=\operatorname{Spec}(R)$, 
the resulting base-change $X=\mathrm{Bl}_{Z}(Y) \times_{Y^{\prime}} S$ yields a proper birational morphism $f: X \rightarrow S$ for which Theorem 1.2 applies, and thus admits a rational pullback $f^{*}: Z^{1}(S) \rightarrow Z^{1}(X)_{\mathbb{Q}}$.

Let me close this section with a standard example that shows that in dimension $d \geq 3$, there are many important $f: X \rightarrow S$ that do not admit a rational pullback: Fix a ground field $k$, and let $R=k[[x, y, u, v]] /(x y-u v)$. By taking partial derivatives, one sees that $S=\operatorname{Spec}(R)$ has an isolated singularity. The ideal $\mathfrak{a}=(x, u)$ defines a prime divisor $D \subset S$. On the blowing-up $X=\operatorname{Proj}(R[\mathfrak{a} T])$ the Cartier divisor defined by the canonical inclusion $\mathscr{O}_{X}(1) \subset \mathscr{O}_{X}$ is the blowing-up of the spectrum of $R / \mathfrak{a}=k[[y, v]]$ at the origin, as one sees by computing $D_{+}(x T)$ and $D_{+}(u T)$. In turn, the exceptional locus $\operatorname{Exc}(X / R)$ coincides with the closed fiber $f^{-1}(z)$, and is is a copy $C=\mathbb{P}^{1}$ of the projective line. In particular, it contains no Cartier divisor. Hence $f: X \rightarrow S$ is a small resolution of singularities. The invertible sheaf $\mathscr{L}=\mathscr{O}_{X}(1)$ for the blowing-up is relatively ample, so $(\mathscr{L} \cdot C)>0$. Since $r=0$, the condition in Definition 1.1 does not hold.

\section{INVERTIBLE M-MATRICES}

After some preparation, we now give a proof for Theorem 1.2. Notation is as in the previous section, in particular $f: X \rightarrow S=\operatorname{Spec}(R)$ is a proper birational morphism, where $R$ is a local noetherian ring that is normal and of dimension $d \geq 2$, and the scheme $X$ is integral and normal. Let us start with a useful general observation:

Lemma 2.1. There is an effective Cartier divisors $D \subset S$ whose strict transform $D^{\prime} \subset X$ intersects each of the exceptional divisors $E_{1}, \ldots, E_{r}$.

Proof. Let $\eta_{i} \in E_{i}$ be the generic points. Then the local rings $\mathscr{O}_{X, \eta_{i}}$ are onedimensional. According to [7], Corollary 1.6, there is a common affine open neighborhood $U \subset X$ for the points $\eta_{1}, \ldots, \eta_{r} \in X$. The complement $A=X \backslash U$, endowed with the reduced scheme structure, is an effective Weil divisor that intersects all $E_{i}$ (confer [8], Chapter II, Proposition 3.1). Its image $f(A) \subset S$ is an effective Weil divisor, with strict transform $A$. Let $\mathfrak{a} \subset R$ be the resulting ideal, and choose some non-zero $\zeta \in \mathfrak{a}$. This defines an effective Cartier divisor $D \subset S$ containing $f(A)$. In turn, the strict transform $D^{\prime} \subset X$ contains $A$, thus intersects each exceptional divisor $E_{i}$.

From now on, we suppose that conditions (i)-(iii) from Theorem 1.2 hold. In particular, each Weil divisor on $X$ is $\mathbb{Q}$-Cartier. Moreover, $N_{1}\left(E_{i}\right) \simeq \mathbb{Z}$. Actually, there is a canonical identification:

Proposition 2.2. For each curve $C \subset E_{i}$, the class $[C] \in N_{1}\left(E_{i}\right)$ is non-zero, and for each further curve $C^{\prime} \subset E_{i}$, the equation $[C]=\mu\left[C^{\prime}\right]$ defines a ratio $\mu \in \mathbb{Q}_{>0}$.

Proof. For this, it suffices to treat the case that both curves $C, C^{\prime}$ are irreducible. Since the proper $k$-scheme $E_{i}$ is connected, there is a sequence of irreducible curves

$$
C=C_{0}, C_{1}, \ldots, C_{n}=C^{\prime}
$$

with $C_{i} \cap C_{i+1}$ non-empty. By induction on $n \geq 0$, it suffices to treat the case that $C \cap C^{\prime}$ is non-empty. Choose an affine open neighborhood $U \subset X$ of some 
intersection point $a \in C \cap C^{\prime}$. The complement $D=X \backslash U$ is an effective Weil divisor, and the intersections $D \cap C$ and $D \cap C^{\prime}$ are both zero-dimensional, hence the intersection numbers $(D \cdot C)$ and $\left(D \cdot C^{\prime}\right)$ are strictly positive. It follows that both classes $[C],\left[C^{\prime}\right]$ are non-zero, and that the ratio $\mu \in \mathbb{Q}$ is strictly positive.

In turn, the one-dimensional vector spaces $N_{1}\left(E_{i}\right)_{\mathbb{Q}}$ are ordered groups whose positive elements are the $\mu[C]$ with $\mu \geq 0$, where $C \subset E_{i}$ is any curve. For each $1 \leq i \leq r$, we now choose some curve $C_{i} \subset E_{i}$, and consider the resulting intersection matrix

$$
\Phi=\left(E_{i} \cdot C_{j}\right) \in \operatorname{Mat}_{r \times r}(\mathbb{Q}) .
$$

Note that this matrix is usually not symmetric, in contrast to the situation in dimension $d=2$. Furthermore, it depends on the choices of curves. It is easy to determine the signs in the intersection matrix, which are actually independent from the chosen curves:

Lemma 2.3. We have $\left(E_{j} \cdot C_{j}\right)<0$ for all $1 \leq j \leq r$, and $\left(E_{i} \cdot C_{j}\right) \geq 0$ for $i \neq j$, with equality if and and only if $E_{i} \cap E_{j}=\varnothing$.

Proof. If $E_{i} \cap E_{j}=\varnothing$, the intersection $E_{i} \cap C_{j}$ is also empty, hence $\left(E_{i} \cdot C_{j}\right)=0$. In the case where $i \neq j$ and $E_{i} \cap E_{j} \neq \varnothing$, we may choose the curve $C_{j} \subset X_{j}$ so that it is not contained in $E_{i}$ but intersects $E_{i}$. In turn, we have $\operatorname{dim}\left(E_{i} \cap C_{j}\right)=0$, and thus $\left(E_{i} \cdot C_{j}\right)>0$. It remains to show $\left(E_{j} \cdot C_{j}\right)<0$. Choose some non-zero non-unit $\zeta \in R$, with Cartier divisor $D \subset S$, and decompose $f^{*}(D)=D^{\prime}+\sum m_{i} E_{i}$, where $D^{\prime}$ is the strict transform and the coefficients are $m_{i}>0$. The inclusion $D^{\prime} \cap E_{j} \subset E_{j}$ is strict, so we may choose the curve $C_{j} \subset E_{j}$ not contained in $D^{\prime}$. Since $f^{*}(D)$ becomes numerically trivial on $C_{j}$, we have

$$
-m_{j}\left(E_{j} \cdot C_{j}\right)=\left(D^{\prime} \cdot C_{j}\right)+\sum_{i \neq j} m_{i}\left(E_{i} \cdot C_{j}\right) .
$$

The intersection numbers on the right are positive, and we are done if at least one is strictly positive. If $r>1$, we find some $i \neq j$ with $E_{i} \cap E_{j} \neq \varnothing$, because $f: X \rightarrow S$ has connected fibers. We may choose $C_{j} \subset E_{j}$ so that it intersects $E_{i}$ but is not contained in $E_{i}$. Thus $\left(E_{i} \cdot C_{j}\right)$ are strictly positive. If $r=1$ we have $j=1$, and the intersection $D^{\prime} \cap E_{1}$ is non-empty. Now we choose $C_{1} \subset E_{1}$ so that it intersects $D^{\prime}$ but is not contained in $D^{\prime}$. In both cases, one intersection number on the right is strictly positive.

Given any real $r \times r$-matrix $A=\left(\alpha_{i j}\right)$ whose off-diagonal entries are $\alpha_{i j} \leq 0$, we may write it in the form $A=s E-B$, for some scalar $s>0$ and some matrix $B=\left(\beta_{i j}\right)$ all whose entries are $\beta_{i j} \geq 0$. Here $E$ denotes the unit matrix. Recall that the spectral radius $\rho(B) \geq 0$ is the maximal length occurring for the complex eigenvalues of $B$. If $\rho(B)<s$ for some scalar $s>0$, the matrix $A$ called an invertible $M$-matrix. Note that if this holds for some $s>0$, it also holds for all $s^{\prime} \geq s$.

The terminology seems to refer to Minkowski, and such matrices have amazing properties. Berman and Plemmons give fifty characterizations of invertible Mmatrices ([2], Chapter 6, Theorem 2.3). One of them is condition $\left(I_{27}\right)$ : There is a column vector ${ }^{t}\left(x_{1}, \ldots, x_{r}\right) \in \mathbb{R}_{>0}^{r}$ with $A x \in \mathbb{R}_{>0}^{r}$. Another one is $\left(N_{38}\right)$ : The inverse $A^{-1}=\left(\lambda_{i j}\right)$ has all entries $\lambda_{i j} \geq 0$. Note that for symmetric matrices, the 
notion boils down to positive-definiteness. We now apply this theory to the negative transpose of our intersection matrices:

Proposition 2.4. The matrix $A=-{ }^{t} \Phi$ is an invertible $M$-matrix. In particular, we have $\operatorname{det}(\Phi) \neq 0$, and all entries of the inverse matrix $\Phi^{-1}$ are negative.

Proof. Suppose $\operatorname{det}(\Phi)=0$. Then there is some non-zero Cartier divisor $\sum m_{i} E_{i}$ such that the resulting invertible sheaf $\mathscr{N}=\mathscr{O}_{X}\left(\sum m_{i} E_{i}\right)$ is numerically trivial on the proper $k$-scheme $E=E_{1} \cup \ldots \cup E_{r}$. After passing to some multiple and renumeration, we may assume that the summands $m_{i} E_{i}$ are Cartier, and that the non-zero coefficients are $m_{1}, \ldots, m_{a}>0$ and $m_{b}, \ldots, m_{r}<0$, for some $1 \leq a<b \leq$ $r+1$. This gives effective Cartier divisors

$$
E^{\prime}=\sum_{i=1}^{a} m_{i} E_{i} \quad \text { and } \quad E^{\prime \prime}=\sum_{i=b}^{r}\left(-m_{i}\right) E_{i}
$$

whose invertible sheaves become numerically equivalent on $E$. We have $\left(E^{\prime} \cdot C_{i}\right) \geq 0$ for all $i>a$, and $\left(E^{\prime \prime} \cdot C_{i}\right) \geq 0$ for all $i<b$. Setting $Y=E^{\prime}$, we see that the invertible sheaf $\mathscr{L}=\mathscr{O}_{X}(Y)$ is nef on each $E_{i}$. On the other hand, the restriction $\mathscr{L}^{\vee} \mid Y=\mathscr{O}_{Y}(-Y)$ is a big invertible sheaf, according to [11], Theorem 1.5. This means that the homogeneous spectrum of the graded ring

$$
R\left(Y, \mathscr{L}^{\vee} \mid Y\right)=\bigoplus_{n \geq 0} H^{0}\left(Y, \mathscr{L}_{Y}^{\otimes-n}\right)
$$

attains the maximal possible dimension $\operatorname{dim}(Y)=d-1$. The notion of big invertible sheaves on integral schemes is common. However, here it is crucial to work with Cutkosky's generalization [4] to arbitrary proper schemes, because our scheme $Y$ usually is reducible and non-reduced. Also note that in loc. cit. we worked with schemes that are proper over an excellent discrete valuation ring, but the argument literally hold true over our excellent local ring $R$.

By [4], Lemma 10.1 combined with Lemma 9.1, there is some irreducible component $E_{j} \subset Y$ such that $\mathscr{L}^{\vee} \mid E_{j}$ is big. In particular, there is some integer $n>0$ and some non-zero global section $\sigma \in H^{0}\left(E_{j}, \mathscr{L}^{\otimes-n} \mid E_{j}\right)$. Write $Z \subset E_{j}$ for the resulting zero-locus, and choose an irreducible curve $C_{j}^{\prime} \subset E_{j}$ not contained in $Z$ but intersecting $Z$. It follows that $\left(\mathscr{L}^{\otimes-n} \cdot C_{j}^{\prime}\right)>0$, contradicting that $\mathscr{L} \mid E_{j}$ is nef. Thus $\operatorname{det}(\Phi) \neq 0$.

To understand the inverse matrix $A^{-1}=-{ }^{t} \Phi^{-1}$, choose an effective Cartier divisor $D \subset S$ as in Lemma 2.1, and write $f^{*}(D)=D^{\prime}+\sum m_{i} E_{i}$. Here $D^{\prime}$ is the strict transform, and all coefficients $m_{i}$ and intersection numbers $\lambda_{j}=\left(D^{\prime} \cdot C_{j}\right)$ are strictly positive. Moreover, $-\left(\sum m_{i} E_{i} \cdot C_{j}\right)=\left(D^{\prime} \cdot C_{j}\right)$ for each $1 \leq j \leq r$. In terms of matrix multiplication, this means

$$
\left(m_{1}, \ldots, m_{r}\right) \cdot(-\Phi)=\left(\lambda_{1}, \ldots, \lambda_{r}\right) .
$$

In turn, $A=-{ }^{t} \Phi$ sends the transpose of $\left(m_{1}, \ldots, m_{r}\right)$ to the transpose $\left(\lambda_{1}, \ldots, \lambda_{r}\right)$, and all entries of these vectors are strictly positive. According to $\left(I_{27}\right)$ in [2], Chapter 6, Theorem 2.3 our $A$ is an invertible M-matrix, and this ensures by $\left(N_{38}\right)$ that the entries in $A^{-1}=-{ }^{t} \Phi^{-1}$ are positive.

Proof of Theorem 1.2. Let $\mathscr{L}$ be an invertible sheaf on $X$ having a global section that does not vanish on any exceptional divisor $E_{1}, \ldots, E_{r}$. The corresponding 
Cartier divisor $D^{\prime} \subset X$ is the strict transform of the Weil divisor $D=f\left(D^{\prime}\right)$. The inclusions $D^{\prime} \cap E_{i} \subset E_{i}$ are strict, so we may choose the curves $C_{i} \subset E_{i}$ so that they are not contained in $D^{\prime}$. In turn, we have $\lambda_{j}=\left(D^{\prime} \cdot C_{j}\right) \geq 0$. Condition (i) ensures that each Weil divisor $E_{i} \subset X$ is $\mathbb{Q}$-Cartier, so we may form the intersection matrix $\Phi=\left(E_{i} \cdot C_{j}\right)$. By Proposition 2.4, this matrix is invertible, and the entries of its inverse are negative. The equation

$$
\left(m_{1} / n, \ldots, m_{r} / n\right)=\left(\lambda_{1}, \ldots, \lambda_{r}\right) \cdot\left(-\Phi^{-1}\right)
$$

defines rational numbers $m_{i} / n \in \mathbb{Q}_{\geq 0}$. In turn, we have

$$
\left(\mathscr{L}^{\otimes n} \cdot C_{j}\right)=\left(n D^{\prime} \cdot C_{j}\right)=n \lambda_{j}=-\sum m_{i}\left(E_{i} \cdot C_{j}\right)=-\left(\sum m_{i} E_{i} \cdot C_{j}\right),
$$

and the $m_{i} / n \in \mathbb{Q}_{\geq 0}$ are the only fractions having this property. By condition (ii), any vertical curve $C \subset f^{-1}(s)$ lies over the closed point $s=z$. If $C$ is contained in $E_{j}$, we have $[C]=\mu_{j}\left[C_{j}\right]$ inside $N_{1}\left(E_{j}\right)$ for some ratio $\mu_{j}>0$, according Proposition 2.2. Note that the latter relies on condition (iii). From equation (4), we infer $\left(\mathscr{L}^{\otimes n} \cdot C\right)=-\left(\sum m_{i} E_{i} \cdot C\right)$. Thus the condition in Definition 1.1 is fulfilled, in other words, our morphism $f: X \rightarrow S$ admits a rational pullback.

\section{REFERENCES}

[1] M. Artin: Some numerical criteria for contractability of curves on algebraic surfaces. Am. J. Math. 84 (1962), 485-496.

[2] A. Berman, R. Plemmons: Nonnegative matrices in the mathematical sciences. Society for Industrial and Applied Mathematics, Philadelphia, PA, 1994.

[3] N. Bourbaki: Algebra II. Chapters 4-7. Springer, Berlin, 1990.

[4] S. Cutkosky: Asymptotic multiplicities of graded families of ideals and linear series. Adv. Math. 264 (2014), 55-113.

[5] T. de Fernex, C. Hacon: Singularities on normal varieties. Compos. Math. 145 (2009), 393-414.

[6] P. Deligne, N. Katz: Groupe de monodromie en géométrie algébrique (SGA 7 II). Springer, Berlin, 1973.

[7] P. Gross: The resolution property of algebraic surfaces. Compos. Math. 148 (2012), 209226.

[8] R. Hartshorne: Ample vector bundles. Publ. Math., Inst. Hautes Étud. Sci. 29 (1966), 63-94.

[9] D. Mumford: The topology of normal singularities of an algebraic surface and a criterion for simplicity. Publ. Math., Inst. Hautes Étud. Sci. 9 (1961), 5-22.

[10] S. Schröer: On non-projective normal surfaces. Manuscr. Math. 100 (1999), 317-321.

[11] S. Schröer: Total separable closure and contractions. Preprint, arXiv:1708.06593.

Mathematisches Institut, Heinrich-Heine-Universität, 40204 Düsseldorf, GerMANY

E-mail address: schroeer@math.uni-duesseldorf.de 